\title{
Papers
}

\section{Survival in treated hypertension: follow up study after two decades}

\author{
Ove K Andersson, Torbjörn Almgren, Bengt Persson, Ola Samuelsson, Thomas Hedner, \\ Lars Wilhelmsen
}

\begin{abstract}
Objective: To compare survival and cause specific mortality in hypertensive men with non-hypertensive men derived from the same random population, and to study mortality and morbidity from cardiovascular diseases in the hypertensive men in relation to effects on cardiovascular risk factors during 22-23 years of follow up.

Design: Prospective, population based observational study.

Subjects and methods: 686 hypertensive men aged 47-55 at screening compared with 6810

non-hypertensive men. The hypertensive men were having stepped care treatment with either $\beta$ adrenergic blocking drugs, thiazide diuretics, or combination treatment. Mortality, morbidity, and adverse effects were registered at yearly examinations and from death certificates.

Main outcome measures: All cause mortality and cause specific mortality.

Results: Treated hypertensive men had significantly impaired probability of total survival as well as survival from coronary heart disease and stroke. All cause mortality as well as coronary heart disease and stroke mortality were very similar in hypertensive men and normotensive men during the first decade, but increased steadily thereafter despite continuous good blood pressure control. Smoking, signs of target organ damage, and high serum cholesterol levels, but not blood pressure at screening, were significantly related to the incidence of coronary heart disease during follow up. In time dependent Cox's regression analysis, the incidence of coronary heart disease was significantly related only to serum cholesterol concentrations in the study. Cancer mortality was almost similar in treated hypertensive men $(61 / 686$, $8.9 \%)$ and non-hypertensive men (732/6810, 10.8\%). Conclusion: Treated hypertensive men had impaired survival and increased mortality from cardiovascular disease compared with non-hypertensive men of similar age. These differences were observed during the second decade of follow up. During an observation period of 22-23 years-about 15000 patient years-hypertensive men receiving diuretics and $\beta$ blockers had no increased risk of cancer or non-cardiovascular disease.
\end{abstract}

\section{Introduction}

Treatment of hypertension prolongs life and prevents or delays congestive heart failure and nephrosclerosis and reduces the incidence of stroke. ${ }^{1-5}$ Antihypertensive treatment has, however, been less effective in preventing coronary heart disease. ${ }^{6}$ It is not clear whether this is due to inadequate blood pressure control, inadequate intervention of other risk factors, negative effects on the risk of cardiovascular disease from antihypertensive drugs, intervention being too late to affect atherosclerosis, or inadequate effect of present drugs on atherosclerosis.

Previous controlled trials on chronic hypertensive disease have been limited by their duration (about 5 years) as a better therapeutic effect has been suggested at later stages of treatment. $^{78}$ If so, important information could be gained from prospective studies of long duration, which would also allow the evaluation of patient characteristics of prognostic importance.

Our population based, prospective study of more than 20 years aimed to analyse the long term mortality in treated hypertensive men compared with nonhypertensive men taking part in the same prevention programme, and to analyse the effects of treatment on mortality from cancer and non-cardiovascular diseases.

\section{Subjects and methods}

\section{Subjects}

The multifactor primary prevention trial started in Gothenburg in 1970. The trial aimed to study whether intervention against smoking, hypercholesterolaemia, and hypertension could reduce the incidence of and mortality from cardiovascular diseases. ${ }^{9}$ The study comprised one third of all men born between 1915 and 1925 except those born in 1922 and 1923. The intervention group comprised 9998 men and two control groups of 10000 men each. The control groups will not be discussed further. The first screening took place between January 1970 and March 1973. Overall, 7495 men took part in the screening (participation rate $75 \%)$.

Hypertension in need of treatment was defined as either a sitting casual systolic blood pressure $>175 \mathrm{~mm} \mathrm{Hg}$ or a diastolic blood pressure $>115 \mathrm{~mm} \mathrm{Hg}$ on two occasions ${ }^{10}$ or a patient already receiving treatment to lower blood pressure because of

\author{
Department of \\ Medicine, \\ Sahlgrenska \\ University Hospital, \\ S-413 45 \\ Gothenburg, \\ Sweden \\ Ove K Andersson, \\ associate professor \\ Torbjörn Almgren, \\ consultant physician \\ Bengt Persson, \\ associate professor \\ Department of \\ Medicine, Section of \\ Nephrology, \\ Sahlgrenska \\ University Hospital \\ Ola Samuelsson, \\ associate professor \\ Department of \\ Clinical \\ Pharmacology, \\ Sahlgrenska \\ University Hospital \\ Thomas Hedner, \\ professor \\ Institute of Heart \\ and Lung Diseases, \\ Section of \\ Preventive \\ Cardiology, \\ Sahlgrenska \\ University Hospital \\ Lars Wilhelmsen, \\ professor \\ Correspondence to: \\ Dr Andersson \\ BMJ 1998;317:167-71
}


hypertension. Of 885 (11.8\%) hypertensive men identified at screening $686(9 \%)$ agreed to have treatment at a special clinic originally formed for the care of such patients. At screening, an afternoon sitting casual reading of their mean blood pressure was 185/ $114 \mathrm{~mm} \mathrm{Hg}$, and at their first visit to the clinic it was $169 / 106 \mathrm{~mm} \mathrm{Hg}$ after 5 minutes of supine rest. Some men needed treatment later; these men were not included in the hypertensive group, but in the following comparisons are included in the normotensive group. ${ }^{9}{ }^{10}$ This diminishes the difference between the two groups.

\section{Methods}

Intervention on risk factors was based upon the concept of multiple risk. For the practical intervention programme it was found feasible, however, to select a discrete cut off point for intervention against each of the risk factors of elevated serum cholesterol concentration, elevated blood pressure, and tobacco smoking, irrespective of the values of the other risk factors in the patient.

Smoking habits were graded on a five point scale: non-smoker (1); ex-smoker (2); smoking 1-14 g of tobacco per day (3); smoking 15-24 g of tobacco per day (4); and smoking $>24 \mathrm{~g}$ of tobacco per day (5). Serum cholesterol concentrations were determined at a laboratory that was regularly standardised. A 12 lead resting electrocardiogram was recorded and coded according to the revised Minnesota code. ${ }^{11}$ Data on mortality were obtained from the national census register. All death certificates were collected and registered. Data on mortality and morbidity from stroke and myocardial infarction were also checked with the local stroke and myocardial infarction registers. ${ }^{12}{ }^{13}$ Two of us (OA and $\mathrm{BP}$ ) also checked all 261 death certificates of the treated hypertensive men with the clinical diagnoses of their patient records and compared these with the codes used in the national census register. Until 1987 we used codes according to the international classification of diseases 8 th revision and thereafter codes according to the international classification of diseases 9 th revision, but for the broad groupings there were no differences between the two. An almost complete (99.8\%) accordance between our coding and that of the national census register was found. Five separate categories were used: ischaemic heart disease (ICD codes 410-414); cerebrovascular disease (430-440); other vascular disease (390-456) excluding the above; cancer (140-207 and 230-239); and all other causes (any other ICD code).

\section{The Gothenburg blood pressure clinic}

The outpatient blood pressure clinic in Gothenburg was set up in 1970 to care for hypertensive patients from the ongoing prevention study, ${ }^{9}$ and to help general practitioners with severely hypertensive patients or those resistant to treatment. ${ }^{10}$ At the first clinic visit a detailed patient history was taken and a physical examination and standardised laboratory tests performed. Blood pressure was measured after 5 minutes of supine rest and after standing for 1 minute. During the study two nurses recorded about $90 \%$ of the blood pressures.

Initial treatment comprising a $\beta$ adrenoceptor blocking agent or a thiazide diuretic was adapted for each patient's needs. If necessary these two drugs were combined to achieve the target blood pressure of $<160 / 95 \mathrm{~mm} \mathrm{Hg}$. In 1988 the target blood pressure was revised to a diastolic blood pressure $<90 \mathrm{~mm} \mathrm{Hg}$ owing to international recommendations. If further drugs were needed to achieve the target blood pressure, a stepped care regimen was followed without a strict protocol. Hence, hydralazine or other drugs, or both were added if necessary. After 10 years, 64\% $(368 / 575)$ of all patients were receiving a $\beta$ adrenoceptor blocking agent; $74 / 575$ (13\%) took this drug alone as single drug treatment. After 15 years the corresponding frequencies were $68 \%(311 / 457)$ and $18 \%(81 / 457)$. Thiazide diuretics were used in $58 \%$ $(\mathrm{n}=334)$ of all patients after 10 years, with $6 \%(\mathrm{n}=35)$ having this drug as single drug treatment. After 15 years the corresponding treatment was $62 \%(283 / 457)$ and $8 \%(\mathrm{n}=37)$.

All hypertensive patients had at least one annual check up. The patient history concerning cardiovascular disease morbidity was updated, and blood pressure measurements and laboratory test data were obtained. Patients who did not keep an appointment were given a new one.

During the first year of follow up $25(3.6 \%)$ of the hypertensive men had stopped attending the clinic, but thereafter the annual withdrawal rate was less than $1 \%$. No patient in the treated hypertensive group was lost to follow up for mortality and morbidity.

\section{Statistical methods}

The data were analysed using PC-SAS (version 6.12, SAS Institute, Cary, NC). ${ }^{15}$ Standard summary statistics were used to illustrate results.

Total and cause specific mortality and cause specific morbidity in the treated hypertensive men (686) were compared with that of the non-hypertensive men who attended the screening examination (6810). Life table analyses of the cumulative survival were performed using the Kaplan-Meier estimate. The log rank test was used to test differences between survival curves.

Mean instudy blood pressure and serum cholesterol measurements were defined as the mean of annual readings after $5,10,15$, and 20 years of follow up or until the end of the follow up period, or the time to drop out, or the last check up visit preceding a cardiovascular complication or death. Patients with a history of a clinically verified myocardial infarction and stroke before entry were excluded from analysis.

The Cox's proportional hazards model was used to test the multivariate correlations between both entry and mean instudy variables and cardiovascular disease. ${ }^{16}$ Among entry characteristics only variables previously shown in this patient series to be significantly associated with cardiovascular disease in multivariate analysis-diastolic blood pressure, serum cholesterol concentration, smoking, and target organ damage based on criteria of the World Health Organisation-were included for analysis. When variables with updated measurements were tested (blood pressure, serum cholesterol concentration, and smoking) the updated covariates proportional hazards model was used. ${ }^{17}$ This model is also known as Cox's time dependent regression model. 


\section{Results}

Table 1 shows the patients' characteristics on entry to the study, and table 2 shows the risk factor changes. Blood pressures were reduced after the first year of follow up, and a further reduction was observed after 5 years. After 10 and 15 years the mean blood pressure was $149 / 89 \mathrm{~mm} \mathrm{Hg}$ and 145/89 $\mathrm{mm} \mathrm{Hg}$ respectively. Serum cholesterol concentrations were reduced and the proportion of smokers decreased from 34\% at baseline to $17 \%$ after 15 years of follow up. Of the 686 hypertensive men, $257(37.4 \%)$ had a significantly higher all cause mortality compared with nonhypertensive men (1992/6810, 29.2\%) (table 3 and fig $1)$. The odds ratio for mortality was $1.6(95 \%$ confidence interval 1.4 to 2.1 ). This increased mortality in hypertensive men was due to a higher incidence of cardiovascular disease $(27.6 \%$ versus $14.2 \%)$ dominated by coronary heart disease $(20.1 \%$ versus $10.3 \%$; odds ratio 1.9, 1.6 to 2.3) (fig 2). Mortality from stroke was also higher, $4.4 \%$ and $1.8 \%$ respectively $(2.1,1.4$ to 2.7). An important observation was that all cause mortality as well as coronary heart disease mortality were similar in hypertensive and normotensive men during the first decade, but this increased steadily despite continuously good blood pressure control.

Mortality from cancer in hypertensive and nonhypertensive men was $8.9 \%$ and $10.8 \%$ respectively.

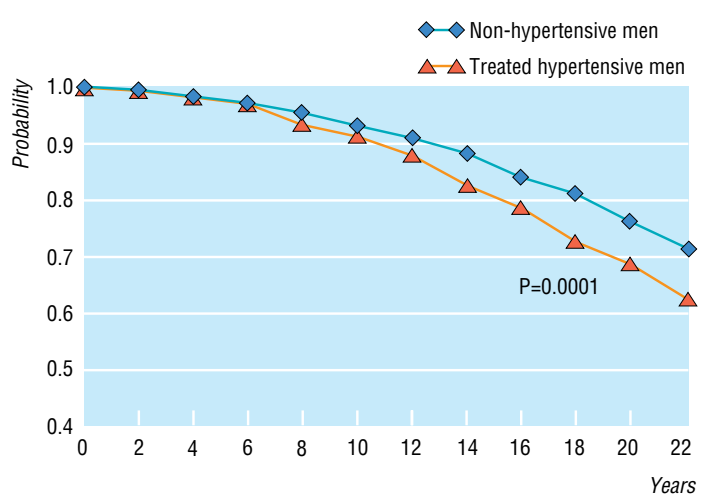

Fig 1 Cumulative probability of survival of 686 men treated for hypertension and 6810 non-hypertensive men in primary prevention study

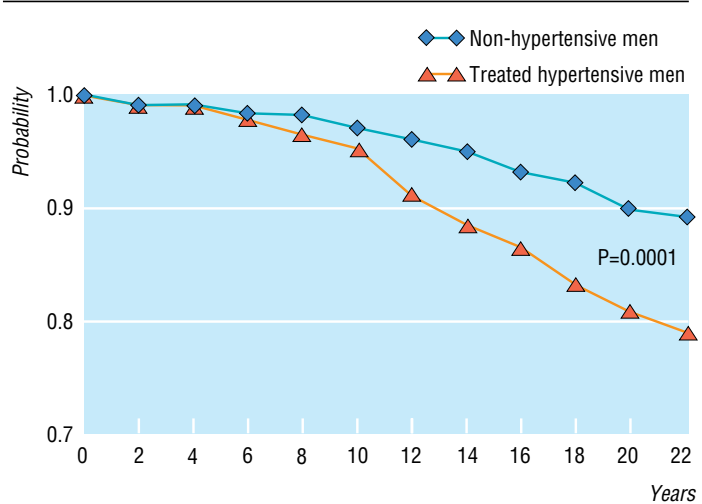

Fig 2 Cumulative probability of survival from coronary artery disease in 686 men with hypertension and 6810 non-hypertensive men in primary prevention study
Table 1 Characteristics at screening of 686 hypertensive men and 6810 non-hypertensive men aged 47-55 years

\begin{tabular}{lcc} 
Characteristic & $\begin{array}{c}\text { Hypertensive men } \\
\text { (mean) (SD) }\end{array}$ & $\begin{array}{c}\text { Non-hypertensive men } \\
\text { (mean) (SD) }\end{array}$ \\
\hline Age (years) & $52.4(2.3)$ & $52.6(2.1)$ \\
\hline Screening blood pressure*: & & $145.5(11.3)$ \\
\hline Systolic $(\mathrm{mm} \mathrm{Hg})$ & $185.3(19.1)$ & $93.4(8.7)$ \\
\hline Diastolic $(\mathrm{mm} \mathrm{Hg})$ & $114.6(12.3)$ & $6.4(1.1)$ \\
\hline Serum cholesterol concentration $(\mathrm{mmol} / \mathrm{l})$ & $6.6(1.2)$ & $2.1(1.0)$ \\
\hline Smoking score $(1-5$ points) $\dagger$ & $2.2(1.1)$ & $76.9(11.3)$ \\
\hline Body weight $(\mathrm{kg})$ & $83.3(12.4)$ & - \\
\hline Height $(\mathrm{cm})$ & $175.4(6.2)$ & - \\
\hline Target organ damage $(\%) \ddagger:$ & & - \\
\hline 1 & 77 & 1.1 \\
\hline 2 & 9 & \\
\hline 3 & 11 & $(5.9)$ \\
\hline Diabetes $(\%)$ & 3.8 & - \\
\hline
\end{tabular}

${ }^{*}$ Sitting blood pressure without previous rest.

$\dagger 1=$ non-smoker; $2=e x-$ smoker; $3=1-14 \mathrm{~g}$ tobacco per day; $4=15-24 \mathrm{~g}$ tobacco per day; $5=>24 \mathrm{~g}$ tobacco per day.

$\ddagger$ According to World Health Organisation staging.

Table 2 Risk factor changes during clinical follow up of 686 hypertensive men

\begin{tabular}{lccccc} 
Follow up & $\begin{array}{c}\text { No of } \\
\text { men }\end{array}$ & $\begin{array}{c}\text { Blood pressure } \\
(\mathbf{m m ~ H g})^{*}\end{array}$ & $\begin{array}{c}\text { Heart rate } \\
\text { (beats/min) }\end{array}$ & $\begin{array}{c}\text { Serum cholesterol } \\
\text { concentration } \\
(\mathbf{m m o l} / \mathbf{l})\end{array}$ & Smoking (\%) \\
\hline Baseline & 686 & $169 / 106$ & 80 & 6.6 & 34 \\
\hline Year: & & & & & \\
\hline 1st & 654 & $158 / 97$ & 72 & 6.5 & 30 \\
\hline 5th & 596 & $148 / 91$ & 67 & 6.2 & -† \\
\hline 10th & 575 & $149 / 89$ & 65 & 6.2 & 27 \\
\hline 15th & 457 & $145 / 89$ & 66 & 6.1 & 17 \\
\hline
\end{tabular}

${ }^{*}$ After 5 minutes of supine rest.

tData not available.

Table 3 Numbers and proportion of deaths in 686 treated hypertensive men and 6810 non-hypertensive men in primary prevention study

\begin{tabular}{lccc} 
& \multicolumn{3}{c}{ No (\%) of deaths } \\
\cline { 2 - 4 } Mortality & Treated hypertensive men & P value & Non-hypertensive men \\
\hline All cause & $257(37.4)$ & 0.001 & $1992(29.2)$ \\
\hline Ischaemic heart disease & $138(20.1)$ & 0.001 & $698(10.3)$ \\
\hline Stroke & $31(4.5)$ & 0.002 & $120(1.8)$ \\
\hline Other cardiovascular disease & $21(3.1)$ & 0.001 & $140(2.1)$ \\
\hline Cancer & $61(8.9)$ & NS & $732(10.8)$ \\
\hline Other causes & $6(0.9)$ & 0.001 & $281(4.1)$ \\
\hline
\end{tabular}

NS=not signficant.

Mortality from all other causes was lower in hypertensive men $(0.9 \%$ versus $4.1 \%)$ (table 3$)$.

In multivariate regression analysis the incidence of coronary heart disease was independently associated with target organ damage (WHO stage 2-3), smoking, and serum cholesterol concentration (table 4). In time dependent Cox's regression analysis only serum cholesterol concentration was significantly predictive of coronary heart disease (table 4).

\section{Discussion}

Our population based study differs from controlled trials of antihypertensive treatment as it has a much longer duration of over 20 years. This study shows that hypertensive men treated long term with the same drugs have an increased mortality compared with nonhypertensive men. This was predominantly observed 
Table 4 Partial regression coefficients, hazard ratios with 95\% confidence intervals, and $P$ values of entry and instudy variables for incidence of coronary heart disease derived by multiple Cox's regression analysis. Analysis was based on 174 cases of first myocardial infarction. Time dependent multiple Cox's regression analysis used in analysis relating instudy variables to future coronary heart disease

\begin{tabular}{lllc} 
Patient characteristic & $\begin{array}{c}\text { Partial regression } \\
\text { coefficient }\end{array}$ & Hazard ratio (95\% Cl) & P value \\
\hline Entry variables: & & & \\
\hline Diastolic blood pressure & 0.09 & $1.01(0.92$ to 1.11$)$ & NS \\
\hline Smoking* & 0.41 & $1.52(1.29$ to 1.58$)$ & $<0.0001$ \\
\hline Serum cholesterol concentration & 0.32 & $1.18(1.13$ to 1.30$)$ & $<0.001$ \\
\hline Target organ damage & 0.78 & $2.11(1.48$ to 3.11$)$ & $<0.0001$ \\
\hline Instudy variables: & & & \\
\hline Diastolic blood pressure & 0.01 & $1.01(0.90$ to 1.21$)$ & NS \\
\hline Smoking & 0.03 & $1.03(0.92$ to 1.19$)$ & NS \\
\hline Serum cholesterol concentration & 0.19 & $1.20(1.08$ to 1.32$)$ & $<0.001$ \\
\hline NS=not significant. & & & \\
${ }^{*}$ Relative number of smokers and non-smokers. & & &
\end{tabular}

during the latter part of follow up despite good blood pressure control.

The higher total mortality rate in treated hypertensive men was due to cardiovascular complications, while mortality from non-cardiovascular causes was lower among treated hypertensive men compared with non-hypertensive men. A carefully structured interventional programme with defined blood pressure targets, regular follow up, and a limited drop out rate does not therefore allow a complete normalisation of cardiovascular risk.

On the basis of the difference in risk factor profile at entry between hypertensive and normotensive men, a certain proportion of hypertensive patients probably already had advanced atherosclerosis and hypertensive target organ damage at the start of follow up. The outcome was therefore not unexpected. The strong association between target organ damage at entry to the study and the incidence of ischaemic heart disease underlies the importance to prognosis of cardiovascular abnormalities present before treatment.

With the exception of target organ damage and smoking at entry to the study, the strongest association with ischaemic heart disease in treated hypertensive men was baseline cholesterol and achieved cholesterol concentrations. Our programme of lifestyle modifications to control hyperlipidaemia was not, however, very effective. Only the lipid lowering agent cholestyramine was available for the major duration of follow up, and it was only prescribed to patients with serum cholesterol concentrations $>7.0 \mathrm{mmol} / \mathrm{l}$. The modest reduction of serum cholesterol concentration to about $6.2 \mathrm{mmol} / \mathrm{l}$ seems therefore suboptimal for a substantial positive effect on coronary atherosclerosis. More recently developed pharmacological methods with the use of statins should have a superior preventive effect. $^{18} 19$

There are several possible explanations for the high long term cardiovascular mortality in the hypertensive men. To what extent this was a consequence of failure to reduce blood pressure to normal is difficult to evaluate. Hence, both systolic and diastolic blood pressures were reduced by $15-20 \%$. If a diastolic blood pressure reduction of about $6 \mathrm{~mm} \mathrm{Hg}$ results in a $36 \%$ reduction of stroke and a $16 \%$ reduction of expected coronary heart disease over about 5 years, ${ }^{6}$ it is conceivable that the blood pressure control achieved in the present study would also be beneficial. However, the reduction in blood pressure did not bring all the patients to strict normotension, and a majority of the treated patients remained in the right part of the total blood pressure distribution curve. Coronary heart disease incidence was not related to achieved blood pressure.

It has been suggested that antihypertensive treatment with $\beta$ blockers and thiazide diuretics may increase cardiovascular risk because of induced impairment of metabolism of lipids and glucose..$^{20-22}$ These drugs were used as first line treatment in the present study because they were available when treatment was started in the early 1970 s. Calcium antagonists of the dihydropyridine type were introduced during the later part of follow up as replacement for hydralazine, and angiotensin converting enzyme inhibitors were only used to a minor extent. Drugs related to metabolism have been previously analysed in the present patient series, but there was no evidence of increased risk of complications from elevated triglyceride concentrations or impaired glucose tolerance induced by treatment. ${ }^{23}$ The treatment in our study may seen outdated, but it should be emphasised that newer treatment with calcium blockers and angiotensin converting enzyme inhibitors became increasingly more common only during the later part of follow up. It was during this period, however, that the increased morbidity among the hypertensive men was most evident. To date no study has shown the effects of more modern treatment on hard end points.

An association between high blood pressure and several metabolic variables, as well as disturbances of the fibrinolytic system, is well known. ${ }^{24-26}$ These abnormalities, which have important prognostic importance in the general population, are not favourably influenced by the antihypertensive drugs available at the time of the present study. Several of these abnormalities, in addition to high blood pressure, may be caused by a common mechanism that is not influenced by such treatment. Whether newer drugs with other modes of action will be superior is presently not known.

Key messages

- Hypertension is a prevalent (10-20\%) and important risk factor for cardiovascular disease.

- In controlled trials over 3-5 years drug treatment for hypertension prevents these complications, but little is known about long term prognosis

- During 20-22 years treated hypertensive men had a significantly increased mortality, especially from coronary heart disease, compared with non-hypertensive men from the same population

- The high incidence of myocardial infarction was related to organ damage, smoking, and cholesterol at the time of entry to the study, and to achieved serum cholesterol concentrations during follow up

- The poor prognosis for mortality from coronary heart disease is dependent upon strict monitoring of serum cholesterol concentrations 
It is possible that the preventive effect of lowering blood pressure was less than expected because treatment was started too late, especially as target organ damage (WHO stage 2-3 at the start of intervention) strongly increased the risk of ischaemic heart disease.

\section{Conclusion}

Our study of a representative group of hypertensive men showed that despite treatment they had a significantly higher long term mortality from cardiovascular diseases than normotensive men. Part of the risk is irreducible with the treatment regimens available at the time of the study, and may be related to cardiovascular damage and risk factors present before established hypertension was diagnosed. During treatment only an elevated serum cholesterol concentration was predictive of coronary heart disease, and high blood pressure levels were associated with an increased risk of stroke.

Contributors: The primary prevention trial in Gothenburg was initiated and designed by LW and Professor Gösta Tibblin. LW was responsible for the study. The present study was designed, conducted, and supervised in all parts by OKA, who will also act as guarantor for the paper. The other authors took an active part in the analyses and interpretation of the data and in writing the paper. We also thank professor Göran Berglund for his previous efforts when initially organising the outpatient clinic for hypertension management at Sahlgrenska University Hospital and our head nurse Ann-Louise Eriksson who followed the patients from the start of the study.

Funding: The Swedish Medical Research Council, the Swedish Heart and Lung Foundation, the Bank of Sweden tercentenary fund, the Knut and Alice Wallenberg Foundation, and the Gothenburg Medical Association.

Conflict of interest: None.

1 Sokolow M, Perloff D. The prognosis of essential hypertension treated conservatively. Circulation 1961;23:697-713.

2 Veterans Administration Cooperative. Study group on antihypertensive agents: effects of treatment on morbidity in hypertension: II. Results in patients with diastolic blood pressure averaging 90 through $114 \mathrm{~mm} \mathrm{Hg}$ JAMA 1970:213:1143-52.

3 Smirk FH. The prognosis of untreated and of treated hypertension and advantages of early treatment. Am Heart J 1972;83:825-40.

4 Bulpitt CJ, Beilin LJ, Clifton P, Coles EC, Dollery CT, Gear JSS. Risk factors for death in treated hypertensive patients. Lancet 1979;ii:134-7.

5 Bulpitt CJ, Beevers GD, Butler A, Coles EC, Hunt D, Munro-Faure AD, et al. The survival of treated hypertensive patients and their causes of death: a report from the DHSS hypertensive care computing project (DHCCP). J Hypertension 1986;4:93-100.

6 Collins R, Peto R, MacMahon S, Herbert P, Fiebach NH, Eberlein KA, et al. Blood pressure, stroke, and coronary heart disease. Part 2, short-term reductions in blood pressure: overview of randomised drug trials in their epidemiological context. Lancet 1990;335:827-38.

7 Chobanian AV. Have long-term benefits of antihypertensive therapy been underestimated? Provocative findings from the Framingham heart study. Circulation 1996;93:638-40.

8 Sytkowski PA, D'Agostino RB, Belanger AJ, Kannel WB. Secular trends in long-term sustained hypertension, long-term treatment, and cardiovascular mortality. The Framingham heart study. Circulation 1996;93:697cular 703 .

9 Wilhelmsen L, Berglund G, Elmfeldt D, Tibblin G, Wedel H, Pennert K, et al. The multifactor primary prevention trial in Göteborg, Sweden. Eur Heart J 1986;7:279-88.
10 Andersson OK, Berglund G, Hansson L, Sannerstedt R, Sivertsson R, Wikstrand J, et al. Organization and efficacy of an outpatien hypertension clinic. Acta Med Scand 1978;203:391-8.

11 Rose GA, Blackburn $\mathrm{H}$. Classification of the electrocardiogram minne-sota code,' cardiovascular survey methods. Geneva: World Health Organisation monograph series 1968;56:98-154.

12 Harmsen P, Tibblin G. A stroke register in Göteborg, Sweden. Acta Med Scand 1972;191:463-70.

13 Elmfeldt D, Wilhelmsen L, Tibblin G, Vedin JA, Wilhelmsson C, Bengtsson C. Registration of myocardial infarction in the city of Göteborg, Sweden: a community study.J Chron Dis 1975;28:173-86.

14 Ray AA. SAS user's guide: basics. Cary, NC; SAS Institute, 1982.

15 SAS/STAT software. The PHREG procedure. Cary, NC; SAS Institute, 1991. (Technical report.)

16 Cox DR. Regression models and life tables (with discussion). JR Stat Soc 1972:34:187-220

17 Altman DG, DeStavola BL. Practical problems in fitting a proportional hazards model to data with updated measurements of the covariates. Stat Med 1994;13:301-41.

18 The Scandinavian Simvastatin Survival Study Group. Randomised trial of cholesterol lowering in 4444 patients with coronary heart disease: the Scandinavian simvastatin survival study (4S). Lancet 1994:344:1383-9.

19 Shepherd J, Cobbe SM, Ford I, Isles CG, Lorimer AR, Macfarlane PW. Prevention of coronary heart disease with pravastatin in men with hypercholesterolemia. N Engl J Med 1995;333:1301-7.

20 Grimm RH, Leon AS, Hunninghake DB, Lenz K, Hannan P, Blackburn $\mathrm{H}$. Effects of thiazide diuretics on plasma lipids and lipoproteins in mildly hypertensive patients: a double blind controlled trial. Ann Intern Med 1981;94:7-11.

21 Day JL, Metcalfe J, Simpson CN. Adrenergic mechanism in control of plasma lipid concentrations. BMJ 1982;284:1145-8.

22 Leren P, Foss PO, Helgeland A, Hjermann I, Holme I, Lund-Larsen PG Effect of propranolol and prazosin on blood lipids. The Oslo study. Lancet 1980;ii:4-6.

23 Samuelsson O, Pennert K, Andersson OK, Berglund G, Hedner T, Persson B, et al. Diabetes mellitus and raised serum triglyceride concentration in treated hypertension-are they of prognostic importance? BMJ 1996:313:660-3

24 Berglund G, Larsson B, Andersson OK, Larsson O, Svärdsudd K, Björntorp P, et al. Body composition and glucose metabolism in hypertensive middle-aged males. Acta Med Scand 1976;200:163-9.

25 Reaven GM. Role of insulin resistance in human disease. Diabetes 1988;38:1595-1607.

26 Eriksson H, Welin L, Wilhelmsen L, Larsson B, Ohlson LO, Svärdsudd K, et al. Metabolic disturbances in hypertension: results from the population study "men born in 1913." J Intern Med 1992;232:389-95.

(Accepted 30 March 1998)

\section{Corrections}

Diagnosis of Creutzfeldt-Jakob disease by measurement of $S 100$ protein in serum: prospective case-control study

An authors' error occurred in this paper by Markus Otto and colleagues (21 February, pp 577-82). In table 4 the median values of serum concentrations of S100 protein were incorrect for some diagnoses. For the diagnosis of multi-infarct dementia the median value should have been 104 [not 393]; the median value for progressive dementia should have been 191 [not 529]; the median for amyotrophic lateral sclerosis should have been 56 [not 179]; and the median for chronic encephalopathy should have been 77 [not 47].

Effectiveness of treatments for infantile colic: systematic review An authors' error occurred in this paper by $\mathrm{P}$ L B J Lucassen et al (23 May, pp 1563-9). In the table the first two effect sizes and $95 \%$ confidence intervals should have read $0.05(-0.17$ to 0.27 ) for Evans et $\mathrm{al}^{14}$ and 0.18 ( -0.03 to 0.40$)$ for Lothe et $\mathrm{al}^{15}$ [not 0.05 ( -0.01 to 0.11$)$ and $0.18(0.08$ to 0.28$)$, respectively].

\section{One hundred years ago Special correspondence: Paris}

Dr Gruby, a Parisian celebrity well known for his original manner of treating his patients, has just passed away. He was called in by nearly all the Parisian notabilities, among whom were Liszt, Chopin, Heine, Ambroise Thomas, Alexandre Dumas, father and son. Daudet also consulted Dr Gruby, but was irritated by his original treatment and directions, and refused to follow them. Dr Gruby died at the age of 88 . The circumstances of his death were in keeping with his extraordinary way of living. He became misanthropic, and shut himself up in his room, refusing to see anyone, half opening the door to receive his food. The police commissary, informed of his habits by the neighbours, forcibly opened the doors on November 16th, and found him lying dead on a mattress placed on the floor across the door, which had been bolted. (BMJ 1898;ii:1651) 